\title{
Horizontal Localization in Simulated Unilateral Hearing Loss
}

\author{
Anvarsamarein Parisa ${ }^{1}$, Nazeri Ahmad Reza ${ }^{1}$, Sameni Seyyed Jalal ${ }^{2}$, \\ Kamali Mohammad ${ }^{3}$, and Zarrin Koob Homa ${ }^{1}$ \\ 'Department of Audiology, School of Rehabilitation Sciences, Shahid Beheshti Medical University, Tehran, \\ ${ }^{2}$ Departments of Audiology, ${ }^{3}$ Basic Sciences in Rehabilitation, School of Rehabilitation Sciences, Iran University of Medical Sciences, \\ Tehran, Iran
}

\section{Received July 28,2017 \\ Revised October 25, 2017 \\ Accepted November 21, 2017}

Background and Objectives: The ability to localize a sound source is one of the binaural hearing benefits in a horizontal plane based on interaural time difference and interaural intensity difference. Unilateral or bilateral asymmetric hearing loss will affect binaural hearing and lead to sound locating errors. In this cross sectional analytical descriptive study, the localization error was investigated when participants turned their heads to the sound source with closed eyes and after simulating unilateral hearing loss by placing earplugs inside the right ear canal. Subjects and Methods: This cross sectional analytical descriptive study was carried out on 30 right-handed adults, 22 female and 8 male (average: 25 years, standard deviation: 3.16 ). They were selected with the available random access method. Horizontal localization was evaluated with five speakers located at $0, \pm 30$, and \pm 60 degree azimuths at a 1-meter distance from the examinee. Narrow-band noise signals were delivered at $35 \mathrm{~dB} S \mathrm{SL}$ in two "without earplug" and "with earplug" situations and the results were compared. The study was performed between September and December 2016 in Tehran, Iran. Results: Significant differences were observed in localization errors between the "with earplug" and "without earplug" situations. The localization differences were greater for left-side speakers (-30 and -60 degrees) compared with right-side speakers (+30 and +60 degrees). The differences were more apparent at 4,000 and $6,000 \mathrm{~Hz}$, which confirmed the effect of unilateral simulated hearing loss on interaural latency differences. Conclusions: Simulating hearing loss by using an earplug in one ear (right) increased localization errors at all frequencies. The errors increased at higher frequencies.

J Audiol Otol 2018;22(1):39-44

KEY WORDS: Hearing · Unilateral hearing loss · Earplug.

\section{Introduction}

Localization is a complex peripheral and central skill that uses direction and distance and is formed at three planes: horizontal, vertical and front-back differentiation. This skill is important for spatial processing, simultaneous communication between multiple talkers, driving by car and biking $[1,2]$. Spatial cues, including spectral notches that are produced by the outer ear and torso, are the main factors for vertical localization and front-back differentiation. Binaural cues (interau-

This is an Open Access article distributed under the terms of the Creative Commons Attribution Non-Commercial License (http://creativecommons.org/licenses/by-nc/4.0/) which permits unrestricted non-commercial use, distribution, and reproduction in any medium, provided the original work is properly cited. ral time and intensity differences) are the main cues for horizontal localization. In fact, the combination of these cues creates a specific combined sign for each place in the space used by the listener for horizontal and vertical localization, front-back differentiation, distance perception and externalization [1-5]. Spatial hearing is considered one of the important aspects of human auditory processing abilities which is evaluated in children and elderly people by different tools [3,4]. Interaural time difference (ITD) and interaural level difference (ILD) are crucial factors in horizontal sound localization. According to duplex theory, ITD is involved in low-frequency sounds, although ILD prepares the primary cues for locating high frequencies [2].

It seems that by introducing a short-term auditory depriva- 
tion (wearing an earplug), auditory physiology is modified in different ways [6]. This phenomena have been investigated by acoustic reflex, Auditory Brainstem Response and loudness evaluation in human being [6,7]. Animal studies also have shown the alteration of receptor trafficking at cochlear nucleus synapses after monaural conductive hearing loss [8]. This study tries to simulate unilateral hearing loss in order to evaluate the effect of the intensity difference between two ears on low- and high-frequency perception. In fact, unilateral hearing loss or bilateral asymmetric hearing loss affects the horizontal localization ability, which has not been studied sufficiently in the audiology literature. Knowing about the effect of unilateral hearing loss on localization, seems to be an important task, because of the different similar situations in real life. These situations are observed more in younger population (like unilateral otitis media, single sided hearing loss, etc.).

This present study was designed to determine the effect of hearing asymmetry on localization. An earplug was used for simulating unilateral hearing loss. The localization performance of the individuals was evaluated at different azimuths and frequencies, before and after using the earplug. The localization deviation was then compared at different frequencies, and the importance of frequency in sound localization was investigated. The aim of this study was to define the effect of unilateral earplug utilization in the localization ability of normal hearing individuals at $0, \pm 30$, and \pm 60 degree azimuths at different frequencies.

\section{Subjects and Methods}

\section{Participants}

This cross-sectional analytical descriptive study was carried out on 30 right-handed, normal-hearing adults (air conduction thresholds $\leq 20 \mathrm{~dB} \mathrm{HL}$ ) comprising 22 females and 8 males [mean age: 25 years, standard deviation (SD): 3.16 range: $21-30$ years]. This study was performed between September and December 2016 at the department of Audiology, School of Rehabilitation, Shahid Beheshti Medical University in Tehran, Iran.

This study was approved by Institutaional Ethics Committee of Shahid Beheshti Medical University, Tehran, Iran (IR. SBMU.RETECH.REC.1396.277). Informed consent was ontained from all participants prior to their participation in the study.

\section{Experimental procedure}

The participants were selected with the available random access method according to the inclusion criteria. Inclusion criteria included presence of normal hrearing at 500, 1,000,
2,000, 4,000, and 6,000 Hz, bilaterally (air conduction $<15$ $\mathrm{dB} H L$ ), age range between $18-30$, right handedness, lack of the history of head truma and lack of cervical difficulties. The participants who were not able to do the instructions or those without enough motivation were excluded from this study. Written consent was first received from all participants. To define right-handedness, all participants completed an Edinburg questionnaire. An otoscopic examination was then performed to observe the external auditory canal and to evaluate the possibility of impacted cerumen. Audiometry was performed by a recently calibrated two channel audiometer (Midimate, GN Otometrics Co., Taastrup, Denmark) in the anechoic chamber, and hearing thresholds between 250 and $8,000 \mathrm{~Hz}$ were evaluated. The participants were then enrolled in the localization study. To perform the localization study, an audiometer (Midimate, Gn Otometrics Co.), 5 loudspeakers (Pejvak Ava Co., Tehran, Iran) and an amplifier unit (Pejvak Ava Co.) were utilized. This experiment was set up in a very silent room acoustically prepared for audiology tests. The speakers were placed on a metal arch in a way that one loudspeaker was placed on a zero degree azimuth and the 4 other speakers were placed at \pm 30 and \pm 60 degree azimuths. The chair was placed at the test point, which was exactly at the center of the one meter radius circle. The height of the speaker's base was adjusted so that the center of each speaker's diaphragm remained at the level of the external auditory meatus. A blindfold was used to eliminate the participant's vision during the localization test.

In the next stage, air-conduction audiometry was performed with narrow band noise stimuli centered at frequencies of $500,1,000,2,000,4,000$, and $6,000 \mathrm{~Hz}$ in order to define the threshold shift after occluding the ear canal. Audiometry was conducted two times, once without ear occlusion and the second time with both auditory canals occluded with foam earplugs (Bilsom 303L, Honeywell, Morris Plains, NJ, USA) [9].

After 15 minutes of rest, the horizontal localization of sound was tested with and without ear canal occlusion. Narrow band noise was delivered at $35 \mathrm{~dB}$ SL from speakers situated at $0, \pm 30$, and \pm 60 degree azimuths at a 1-meter distance and without vision help. To begin this stage, a headband with a focal laser lamp was placed on the head of participants, who were instructed to sit in a way that the laser light would be focused on the center of the diaphragm of the 0 degree speaker. Then, the participant was instructed to turn his/her head toward the source of the delivered sound. We performed some educational trials. In this stage of the study, two separate tests were performed with 15 -minute intervals. The first test was localization with open ears, and the second was localization with the right ear occluded by an earplug. 
In each part of the study, 3 stimuli were delivered in a nonsuccessive way from each speaker, and the exact degree on the graduated surface of the arch as confirmed by laser light and the oral answer of the participant ("yes...here") was recorded. Finally, in order to reduce the magnitude of errors the average of 3 sound deliveries from each speaker was calculated for 5 frequencies $(500,1,000,2,000,4,000$, and 6,000 $\mathrm{Hz}$ ) in both non-occluded and occluded states.

Notably, in this study 3 stimuli were delivered from each speaker at each frequency in each of two conditions (occluded and non-occluded) for each listener ( 5 frequencies $\times 5$ speakers $\times 3$ stimuli). Therefore, for each participant, 150 stimuli were delivered in total. This study was performed by one observer who was an expert audiologist. No missing value was observed during the study.

\section{Statistical analysis}

The statistical analysis was performed by SPSS software ver. 16 (SPSS Inc., Chicago, IL, USA) and the $p$-value $<0.05$ was considered as significant difference in this study.

\section{Results}

First, in order to define the occlusion-induced threshold shift, the binaural occluded thresholds were subtracted from the non-occluded ones. The averages of threshold shifts at different frequencies are shown in Fig. 1.

To evaluate the threshold shift at different frequencies with respect to the normal distribution of data according to the Kol-
mogorov-Smirnov test, one-way analysis of variance (ANOVA) was utilized, and the results showed that the threshold shifts were not equal at different frequencies $(\mathrm{F}=26.53, p<$ $0.001)$. The threshold shifts increased with the increasing frequency (Fig. 1). With respect to the post hoc test (Bonferroni), we found that the average of the threshold shift at each frequency was not significantly different with adjacent frequencies (calculating the SD at $0.95 \%$ interval), but it was significantly different than other frequencies $(p<0.001)$ (Table 1). These

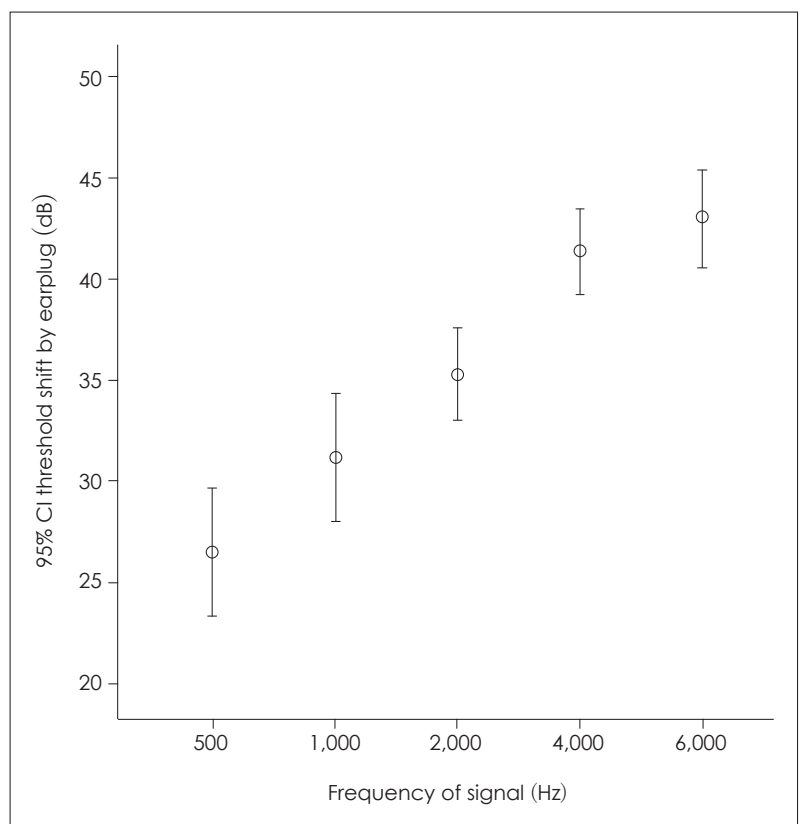

Fig. 1. Average of the threshold shifts after wearing earplug at different frequencies. $\mathrm{Cl}$ : confidence interval.

Table 1. Post hoc test (Bonferroni) results of threshold shifts after earplug use between adjacent frequencies

\begin{tabular}{|c|c|c|c|c|c|c|c|c|c|c|}
\hline \multirow{2}{*}{ Frequency $(\mathrm{Hz})$} & \multicolumn{2}{|c|}{500} & \multicolumn{2}{|c|}{1,000} & \multicolumn{2}{|c|}{2,000} & \multicolumn{2}{|c|}{4,000} & \multicolumn{2}{|c|}{6,000} \\
\hline & SE & Sig & SE & Sig & SE & Sig & SE & Sig & SE & Sig \\
\hline 500 & & & 1.84 & 0.28 & 1.84 & 0.00 & 1.84 & 0.00 & 1.84 & 0.00 \\
\hline 1,000 & 1.84 & 0.28 & & & 1.83 & 0.24 & 1.83 & 0.00 & 1.83 & 0.00 \\
\hline 2,000 & 1.84 & 0.00 & 1.83 & 0.24 & & & 1.83 & 0.01 & 1.83 & 0.00 \\
\hline 4,000 & 1.84 & 0.00 & 1.83 & 0.00 & 1.83 & 0.01 & & & 1.83 & 1.00 \\
\hline 6,000 & 1.84 & 0.00 & 1.83 & 0.00 & 1.83 & 0.00 & 1.83 & 1.00 & & \\
\hline
\end{tabular}

SE: standard error

Table 2. The average and SD of the localization azimuth in the "without earplug" situation for different frequencies and speaker azimuth

\begin{tabular}{|c|c|c|c|c|c|c|c|c|c|c|}
\hline \multirow{3}{*}{ Frequency $(\mathrm{Hz})$} & \multicolumn{10}{|c|}{ Azimuth (degree) } \\
\hline & \multicolumn{2}{|c|}{-60} & \multicolumn{2}{|c|}{-30} & \multicolumn{2}{|c|}{0} & \multicolumn{2}{|c|}{+30} & \multicolumn{2}{|c|}{+60} \\
\hline & Mean & SD & Mean & SD & Mean & SD & Mean & SD & Mean & SD \\
\hline 500 & -58.11 & 1.53 & -31.35 & 2.00 & - & - & 31.28 & 2.17 & 57.52 & 2.23 \\
\hline 1,000 & -57.64 & 2.56 & -31.39 & 1.71 & - & - & 31.49 & 1.91 & 57.93 & 1.80 \\
\hline 2,000 & -59.12 & 2.77 & -31.48 & 1.91 & - & - & 31.90 & 2.30 & 57.28 & 3.80 \\
\hline 4,000 & -57.80 & 2.15 & -29.52 & 11.41 & - & - & 31.28 & 2.23 & 57.79 & 1.93 \\
\hline 6,000 & -58.38 & 1.80 & -31.52 & 1.86 & - & - & 30.75 & 1.92 & 57.94 & 2.00 \\
\hline
\end{tabular}

SD: standard deviation 
Table 3. The average and SD of the localization azimuth in the "with earplug" situation for different frequencies and speaker azimuth

\begin{tabular}{|c|c|c|c|c|c|c|c|c|c|c|}
\hline \multirow{3}{*}{ Frequency $(\mathrm{Hz})$} & \multicolumn{10}{|c|}{ Azimuth (degree) } \\
\hline & \multicolumn{2}{|c|}{-60} & \multicolumn{2}{|c|}{-30} & \multicolumn{2}{|c|}{0} & \multicolumn{2}{|c|}{+30} & \multicolumn{2}{|c|}{+60} \\
\hline & Mean & SD & Mean & SD & Mean & SD & Mean & SD & Mean & SD \\
\hline 500 & -54.04 & 6.63 & -31.36 & 4.10 & -13.91 & 12.67 & 1.07 & 24.56 & 9.18 & 33.40 \\
\hline 1,000 & -49.78 & 8.86 & -30.27 & 8.61 & -14.58 & 14.99 & -19.45 & 31.88 & -12.61 & 34.24 \\
\hline 2,000 & -54.88 & 4.74 & -34.35 & 14.84 & -30.75 & 8.58 & -36.13 & 15.78 & -34.66 & 17.39 \\
\hline 4,000 & -57.54 & 3.24 & -38.40 & 9.82 & -37.07 & 9.71 & -36.80 & 10.57 & -35.55 & 20.49 \\
\hline 6,000 & -57.98 & 3.82 & -36.88 & 8.58 & -31.59 & 16.31 & -38.07 & 13.25 & -38.78 & 18.84 \\
\hline
\end{tabular}

SD: standard deviation

results indicated that earplug efficiency in noise reduction was greater at higher frequencies; by using it unilaterally, interaural latency difference will seemingly be induced, especially at 4 $\mathrm{kHz}$ and $6 \mathrm{kHz}$.

The localization azimuth was then evaluated separately for "with earplug" and "without earplug" situations at different frequencies. The average and SD for the localization azimuth is shown for without earplug situation in Table 2. Obviously, there is no data for the 0-degree column since the participants had no head movement when the signal was delivered from the 0 degree azimuth.

The average and SD for localization azimuth in the right-ear occluded situation at a all azimuths is shown in Table 3. With respect to the normal distribution of data (Kolmogorov-Smirnov), a paired $\mathrm{T}$ test was performed to compare the localization azimuth in two situations. The results showed that for all frequencies at +30 and -60 degrees, the localization azimuth in the occluded right ear situation is significantly less than the nonoccluded situation $(p<0.001)$. At -30 degrees azimuth, there was no significant difference between frequencies of 500 , 1,000 , and $2,000 \mathrm{~Hz}$ (95\% confidence interval) between two situations, but at frequencies of $4,000 \mathrm{~Hz}$ and $6,000 \mathrm{~Hz}$, the localization azimuth is more negative in a right-occluded situation $(p=0.01,400 \mathrm{~Hz} / p=0.01,6,000 \mathrm{~Hz})$. At -60 degrees azimuth, the localization azimuth in the right-ear occluded situation is different from the non-occluded situation for 500, 1,000 , and $2,000 \mathrm{~Hz}(p=0.003)$, and there is no difference at 4,000 $\mathrm{Hz}$ and 6,000 Hz. At 0 degree azimuth, one sample T test showed that the average of localization azimuth in rightear occluded situations is more negative at all frequencies and is increased by the frequency rising from $500 \mathrm{~Hz}$ to $2,000 \mathrm{~Hz}$ ( $p<$ $0.001)$.

To evaluate the role of speaker azimuth and stimulus frequency in the localization function, a localization difference variable was used in two situations to evaluate the effect frequency and azimuth. With respect to the normal distribution of data (Kolmogorov-Smirnov), a one-way ANOVA was utilized. The results showed that the magnitude of deviation was dependent on the stimulus frequency and speaker azimuth. Fig. 2

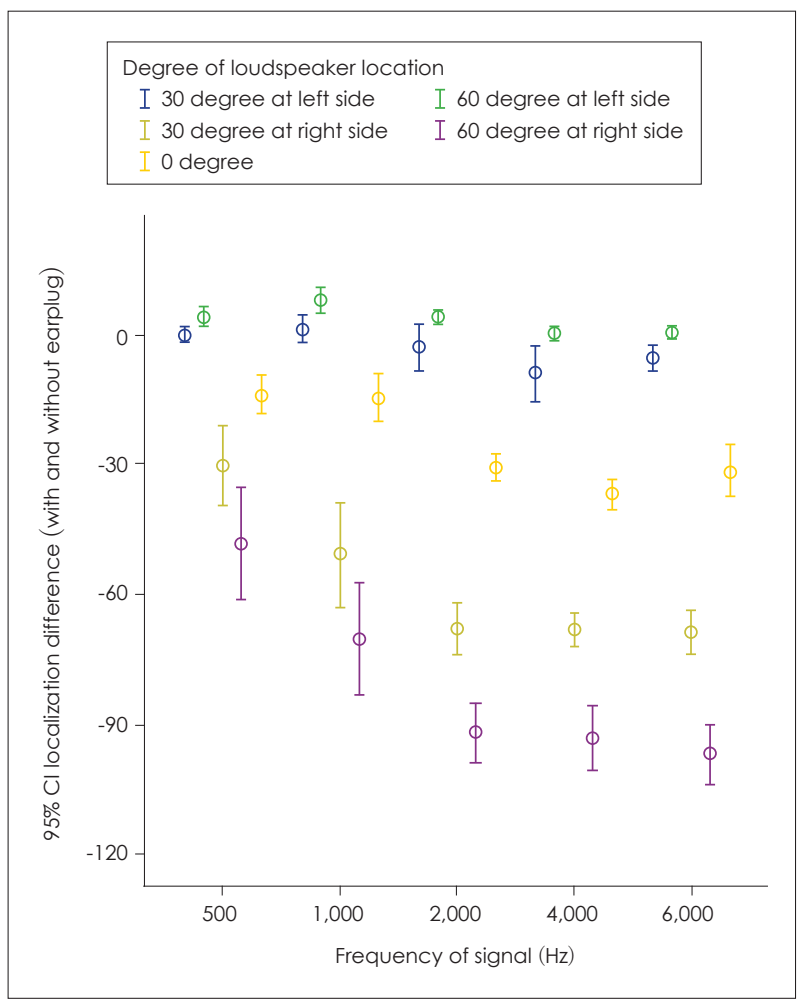

Fig. 2. The average and variability range of localization difference with/without earplugs versus the stimulus frequency at different azimuths. Cl: confidence interval.

shows the average and variability range of localization difference in with- and without-earplug situations in relation to the frequency at different azimuths. The localization difference with and without the earplug is increased from 0 degree to speakers located on the right side, which might be due to right ear canal occlusion and its effect on horizontal localization.

\section{Discussion}

Sound source localization is one of the most crucial abilities of auditory system and is necessary to implement and interpret spatial cues [10-12]. Spatial cues constitute factors such as frequency, sound intensity and the distance between the sound source and listener [13]. ITD and intra-aural intensity 
difference, in relation to the distance between the right and left ear and the azimuth of the sound source to the head, are important for horizontal sound localization [2].

This study targets the horizontal aspect of localization, which is related to ITD and ILD. Effected horizontal localization and associated deviation in sound source locating are expected in unilateral hearing loss and bilateral asymmetric hearing loss. Unilateral hearing loss is simulated in this study by inserting a foam earplug into the right auditory canal.

The magnitude of the threshold shift was not equal at different frequencies. The threshold shift ranged from $26.5 \mathrm{~dB}$ at $500 \mathrm{~Hz}$ to $43 \mathrm{~dB}$ at $6,000 \mathrm{~Hz}$. Although according to post hoc results, the threshold shift of $1,000 \mathrm{~Hz}$ compared with adjacent frequencies i.e., $500 \mathrm{~Hz}(26.50)$ and $2,000 \mathrm{~Hz}(35.33 \mathrm{~dB})$ were not significant at a $95 \%$ confidence interval but were numerically different. The threshold shift was significant for nonadjacent frequencies.

When comparing the present study data for earplug 303L and the producer company data, the average sound attenuation is less in the present study at 500 and $1,000 \mathrm{~Hz}$ frequencies, but for $2,000 \mathrm{~Hz}$ and 4,000 Hz, we found similar results.

Regarding sound localization, for the "without earplug" situation, the average azimuth was very close to the speaker's azimuth. For right-side speakers, the maximum deviation for the +30 degree azimuth was +1.90 and for the +60 degree azimuth was $-2.72(2,000 \mathrm{~Hz})$. The cause of this amount of deviation is head and neck movement error, especially for the +60 degree azimuth. For the left side speakers, the maximum average deviation for the -30 degree azimuth was -1.52 $(400 \mathrm{~Hz})$ and for the -60 degree azimuth was $+2.36(1,000$ $\mathrm{Hz}$ ), which was also due to head and neck movement error. The SD of data is lower for Table 2 (without earplug) compared with Table 3 (right ear occluded with earplug), which confirmed the better localization accuracy of participants with true binaural hearing.

The effect of ear canal occlusion by earplug was not robust for left-side speakers (Table 2), although for the zero azimuth and right-side speakers $(+30,+60$ degree azimuth), the localization differences were increased by frequency increments, which could be explained by the effect of ILD, especially at higher frequencies. These results are similar to those of Humes, et al. [10] They investigated the sound localization skills for normal adults, normal children and children with unilateral hearing loss in the horizontal plane and found the apparent effect of frequency in all groups. In addition, the children with unilateral hearing loss showed reduced localization ability compared with adults at all conditions between 500 and 3,000 $\mathrm{Hz}$. The greatest localization error was at 3,000 Hz. Gustafson and Hamill [14], compared right- and left-ear occlu- sion with an earplug and concluded that, for 3,000 Hz warble tone, right-side occlusion has a greater effect on localization ability. A 64 degree of error was observed on the right side compared to a 58 degree of error on left side. By analyzing Table 1, 3 in this present study, the localization error for $\mathrm{a}+30$ degree speaker with right side occlusion at $200 \mathrm{~Hz}$ was 68.03 degrees and at $4,000 \mathrm{~Hz}$ was 68.08 degrees, which is close to the 64 degree error in the Gustafson and Hamil [14] study.

It is clear that unilateral hearing loss reduces the horizontal localization ability in both sensorineural involvement [15] and conductive impairment [11,16-20]. Häusler, et al. [21] found that wide-band signal localization is not affected with long-term conductive hearing loss lower than $25 \mathrm{~dB}$. They concluded that the results of localization by earplug utilization are similar to those in people who suffer from conductive hearing impairment with more than a $35 \mathrm{~dB}$ hearing threshold at high frequencies. In simulated hearing loss studies, the localization skill has improved over a long period of time [18]. This has been true for unilateral simulated hearing loss individuals who have passed a localization training program [18,20].

In a study by Irving and Moore [22], sound localization training was investigated with and without unilateral occlusion (with earplug). They concluded that learning is able to improve localization gradually; they observed that, after removing the earplug, localization improved to the initial state. The positive effect of localization training is studied in even unilateral severe to profound hearing impaired people $[6,23]$.

\section{Limitations}

There were some limitations for conducting the present study. The Lack of an instrument for divided projection of signals into 5 speakers. The study was long lasting and the participants became tired. The lack of a sensitive laser sensor for automatic detection of the head movement localization.

\section{Clinical application}

According to the results of this present study, unilateral conductive hearing loss degraded the horizontal localization ability which is part of spatial processing. Therefore treating the even small amounts of unilateral conductive hearing loss is critical to prevent spatial processing disabilities.

In conclusion, according to the results of this study, unilateral hearing loss or bilateral asymmetric hearing loss can cause ILD deviation and conclusive localization error in the horizontal plane, especially at higher frequencies. The direction of the sound source is also very important: in situations in which the sound source and the better ear have the same direction, less localization error is observed. Therefore, in unilateral hearing loss, localization error in the horizontal plane will increase. 


\section{Acknowledgments}

This article is extracted from a Master of Science thesis in the Department of Audiology, School of Rehabilitation, Shahid Beheshti Medical University. We appreciate Mrs. Marzieh Sharifian, the head of the Audiology Department, for her assistance and for allowing us to use an acoustic room during the project. We appreciate the Pejvak Ava Company and its general manager Mr. Salek for designing and producing the special amplifier that was able to divide and deliver different test signals to speakers and for his assistance in system calibrations. We appreciate all the students and staff of the School of Rehabilitation for their diligent participation in our project.

\section{Conflicts of interest}

The authors have no financial conflicts of interest.

\section{REFERENCES}

1) Gelfand SA. Chapter 13. Binaural and spatial hearing. In: Gelfand SA, editor. Hearing: an introduction to psychological and physiological acoustics. 5th ed. Boca Raton, FL: CRC Press;2004. p.231-56.

2) Culling JF, Akeroyd MA. Spatial hearing. In: Plack CJ, editor. The Oxford handbook of auditory science: volume 3. Hearing. 1st ed. London: Oxford University Press;2010.

3) Lotfi Y, Nazeri AR, Asgari A, Moosavi A, Bakhshi E. Iranian version of speech, spatial, and qualities of hearing scale: a psychometric study. Acta Med Iran 2016;54:756-64.

4) Delphi M, Zamiri Abdolahi F, Tyler R, Bakhit M, Saki N, Nazeri AR. Validity and reliability of the Persian version of spatial hearing questionnaire. Med J Islam Repub Iran 2015;29:231.

5) Middlebrooks JC, Green DM. Sound localization by human listeners. Annu Rev Psychol 1991;42:135-59.

6) Brotherton H, Plack CJ, Schaette R, Munro KJ. Time course and frequency specificity of sub-cortical plasticity in adults following acute unilateral deprivation. Hear Res 2016;341:210-9.

7) Brotherton H, Plack CJ, Schaette R, Munro KJ. Using acoustic reflex threshold, auditory brainstem response and loudness judgments to investigate changes in neural gain following acute unilateral deprivation in normal hearing adults. Hear Res 2017;345:88-95.
8) Wang H, Yin K, Rogers K, Miralles C, De Blas AL, Rubio ME. Monaural conductive hearing loss alters the expression of the GluA3 AMPA and glycine receptor $\alpha 1$ subunits in bushy and fusiform cells of the cochlear nucleus. Neuroscience 2011;199:438-51.

9) Bilson 303L, industrial safety [cited 2017 Dec 20]. Available from: https://www.honeywellsafety.com.

10) Humes LE, Allen SK, Bess FH. Horizontal sound localization skills of unilaterally hearing-impaired children. Audiology 1980;19:508-18.

11) Bess FH, Tharpe AM. Unilateral hearing impairment in children. Pediatrics 1984;74:206-16.

12) Sabin AT, Macpherson EA, Middlebrooks JC. Human sound localization at near-threshold levels. Hear Res 2005;199:124-34.

13) Florentine M. Relation between lateralization and loudness in asymmetrical hearing losses. J Am Audiol Soc 1976;1:243-51.

14) Gustafson TJ, Hamill TA. Differences in localization ability in cases of right versus left unilateral simulated conductive hearing loss. J Am Acad Audiol 1995;6:124-8.

15) Bess FH, Tharpe AM, Gibler AM. Auditory performance of children with unilateral sensorineural hearing loss. Ear Hear 1986;7:20-6.

16) Newton VE. Sound localisation in children with a severe unilateral hearing loss. Audiology 1983;22:189-98.

17) Viehweg R, Campbell RA. Localization difficulty in monaurally impaired listeners. Ann Otol Rhinol Laryngol 1960;69:622-34

18) Bauer RW, Matuzsa JL, Blackmer RF. Noise localization after unilateral attenuation. J Acoust Soc Am 1966;40:441-4.

19) Gatehouse RW. Further research in localization of sound by completely monaural subjects. J Auditory Res 1976;16:265-73.

20) Fisher HG, Freedman SJ. Localization of sound during simulated unilateral conductive hearing loss. Acta Otolaryngol 1968;66:213-20.

21) Häusler R, Colburn S, Marr E. Sound localization in subjects with impaired hearing. Spatial-discrimination and interaural-discrimination tests. Acta Otolaryngol Suppl 1983;400:1-62.

22) Irving $S$, Moore DR. Training sound localization in normal hearing listeners with and without a unilateral ear plug. Hear Res 2011;280: 100-8.

23) Firszt JB, Reeder RM, Dwyer NY, Burton H, Holden LK. Localization training results in individuals with unilateral severe to profound hearing loss. Hear Res 2015;319:48-55. 